\title{
Desenvolvimento Distribuído de Software: um Modelo de Classificação dos Níveis de Dispersão dos Stakeholders
}

Rafael Prikladnicki ${ }^{1}$, Leandro Lopes ${ }^{1}$, Jorge Luis N. Audy ${ }^{1}$, Roberto Evaristo

${ }^{1}$ Faculdade de Informática - PUCRS

90.619-900 - Porto Alegre - RS - Brasil

2 University of Illinois at Chicago - College of Business Administration 601 S. Morgan Street MC 294, Chicago, IL 60607, United States

\{rafael, lteixeira, audy\}@inf.pucrs.br, evaristo@uic.edu

Resumo. O objetivo deste artigo é propor um modelo de classificação dos niveis de dispersão dos stakeholders envolvidos em projetos de desenvolvimento distribuido de software. Para isto, propõem-se algun critérios para a definição dos niveis de dispersão. O modelo de classificação proposto é parte resultante de uma dissertação de mestrado e foi aplicado em dois estudos de caso, cujos resultados são apresentados e discutidos. Estes estudos de caso compreendem uma organização multinacional que desenvolve projetos com equipes distribuidas em diversos paises em mais de um continente. Esta pesquisa é caracterizada como exploratória, com estudo de caso como método de pesquisa principal. Como resultado, apresenta-se o modelo proposto, discutindo suas vantagens e a sua representação.

\section{Introdução}

Nos últimos anos pode-se perceber um grande avanço em direção à globalização dos negócios, em particular nos negócios relacionados com um intenso investimento na tecnologia de desenvolvimento de software. Dada esta evolução, sabe-se que o software tem se tornado um componente vital para quase todos os negócios. Neste sentido, para as organizações que buscam sucesso é clara a necessidade do uso da Tecnologia da Informação (TI) como diferencial competitivo [1]. A globalização da economia, a crescente competitividade do meio empresarial, a forte pressão por redução de custos e o papel cada vez mais estratégico das Tecnologias da Informação nas organizações têm estimulado o desenvolvimento distribuído de software (DDS) em escala mundial. Neste sentido, as empresas buscam também maior qualidade no processo de desenvolvimento de software e a possibilidade de dispor de recursos em âmbito global [2]

Hoje em dia, é cada vez mais significativo o número de empresas que estão distribuindo seus processos de desenvolvimento de software ao redor do mundo. E este ambiente criou uma nova classe de problemas a serem resolvidas pelos pesquisadores na área de desenvolvimento de software, centrada no DDS. Entre as dificuldades existentes em projetos de DDS, pode-se citar as diferenças culturais entre os integrantes das equipes distribuídas, a aquisição de confiança, tecnologia de comunicação, processo de desenvolvimento, alocação de tarefas, entre outros. Muitas vezes estas dificuldades ocorrem, pois não existe uma identificação correta das pessoas envolvidas nos projetos (tipos de atores) e a respectiva localização de cada uma (niveis de dispersão). 
Neste sentido, este artigo tem por objetivo propor um modelo para classificer os níveis de dispersão dos atores (stakeholders) ${ }^{l}$ envolvidos em projetos de DDS, seguind critérios previamente estabelecidos. Para isto, avança-se no sentido de analisar critérios de definição dos níveis de dispersão em um projeto de DDS e propõe-se us classificação dos niveis de distribuição. A base empírica deste estudo envolve uma organização multinacional que desenvolve projetos com equipes distribuídas ena diversos países e em mais de um continente.

Este artigo tem a seguinte estrutura: a seção 2 apresenta o referencial teóric utilizado; a seção 3 discute as dimensões do DDS; a seção 4 descreve o método de pesquisa utilizado; a seção 5 apresenta o modelo proposto; a seção 6 apresenta 0 estuć de caso e os resultados encontrados; na seção 7 apresentam-se as considerações fin direcionando para estudos futuros e limitações; e finalmente, a seção 8 apresenta as referências bibliográficas.

\section{Desenvolvimento Distribuído de Software (DDS)}

Observou-se na última década um grande investimento na conversão de mercados nacionais em mercados globais, criando novas formas de competicão e colaboração entre os países [3]. Entretanto, o mercado global de software vinha passando por diversas crises. Por um lado, um grande número de falhas em projetos. De outro, uma crescente demanda, atingida pela escassez de recursos capacitados. Nesse ambiente, muitas organizações encontraram no Desenvolvimento Distribuído de Software (DDS) uma alternativa, experimentando o desenvolvimento de software com equipes geograficamente distantes entre si $[4,5,6]$.

O DDS tem sido caracterizado principalmente pela colaboração e cooperação entre departamentos de organizações e pela criação de grupos de pessoas que trabalham em conjunto, mas estão localizados em cidades ou países diferentes. Dependendo da maturidade, cada organização tem a sua própria estratégia de distribuição, relacionada com o tipo de atividade a ser realizada pelas equipes distribuídas (codificação, testes, especificação). Segundo Carmel [4], o DDS tem está causando um grande impacto não apenas no mercado propriamente dito, mas na maneira como os produtos de software têm sido modelados, construídos, testados e entregues para os clientes. Além disso, 0 autor destaca que trabalhar com DDS é um dos maiores desafios que o atual ambiente de negócios apresenta do ponto de vista do processo de desenvolvimento de software.

Diversos fatores têm contribuído para o crescimento do DDS, entre eles:

- Custo mais baixo e disponibilidade de mão de obra;

- Evolução das ferramentas de desenvolvimento;

- A necessidade de possuir recursos globais para utilizar a qualquer hora;

- As vantagens de estar perto do mercado local;

- A formação de equipes virtuais para explorar as oportunidades de mercado;

- A pressão para o desenvolvimento time-to-market, utilizando as vantagens proporcionadas pelo fuso horário diferente, no desenvolvimento conhecido como round-the-clock, ou seja, 0 desenvolvimento quase que contínuo.

Um stakeholder é qualquer pessoa ou representante de uma organização que possua um stake-um grande interesse - no resultado de um projeto [3].
Neste sentido, o DDS tem atraído um grande número de pesquisas na area de Software $[5,6,7]$. Os engenheiros de software têm reconhecido a grande Engenlaria de trabalho no seu dia-a-dia e estão em busca de modelos influencia desta ne com equipes geograficamente distantes. que facilitem o desentor tes pesquisadores têm feito no intuito de entender os que freqüentes os esforços que os pesquisadores tem feito no intito de entender os são fre permitem organizações multinacionais ou virtuais a obterem sucesso fatores que perás das fronteiras físicas e culturais dos países. Em suma, existe uma trabalhando atrave de software. E o DDS, ao série de problemas e desa dispersão geográfica e dispersão temporal acentuou alguns dos acrescentar fatores desenvolvimento.

desafios existentes e acrescentc

\section{As dimensões do DDS}

A pesquisa realizada por Evaristo et al [7] propõe a existência de algumas eñes no contexto de equipes de projetos distribuídos. Estas dimensões (Figura 1) dimensoes no contexto tipo de auxiliam no entendimento e afetam diretamente na performance destes projetos.

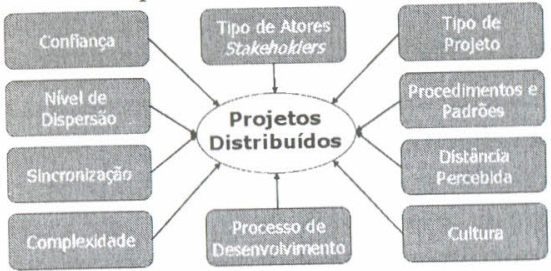

Figura 1 - Dimensões no contexto de projetos distribuídos [7]

Entre as dimensões propostas pelo autor, este artigo irá concentrar-se no estudo rrofundado de duas delas: nivel de dispersão e tipos de atores (stakeholders)

Tipos de Atores (stakeholders): diferentes tipos de grupos de stakeholder existem em cada projeto, cada um com diferentes percepções sobre o projeto. Quanto maior o número de stakeholders envolvidos, maior pode ser a distribuição do projeto.

Nível de Dispersão: a distância física entre os membros de um determinado grupo de stakeholders (equipe de projeto, por exemplo) é chamado de nível de dispersão. Alguns trabalhos exploraram possibilidades de caracterização do nível de dispersão considerando a existência de três atores no desenvolvimento de um projeto (equipe de projeto, clientes e usuários) [8,9], e também considerando as atividades de engenharia de requisitos [10]. Segundo Evaristo et al [7], quanto maior o nível de dispersão, maior é a dificuldade de monitorar o comportamento de diferentes grupos.

3.1 Tipo de atores (stakeholders)

Em um projeto de desenvolvimento de software, podem existir diverso stakeholders (ares), cada um com o seu interesse particular no projeto. Quando abordamos as peculiaridades dos projetos de desenvolvimento distribuído de software, podem existir diferentes tipos de atores.

O estudo realizado por Prikladnicki [9] considerou a existência de três atores principais em projetos de DDS: clientes, usuários e equipe de projeto. Neste caso, a equipe de projeto representava todos os envolvidos no desenvolvimento de um determinado projeto, podendo também ser formada por um conjunto de sub-equipes. Esta equipe envolvia responsáveis pela área de negócios, gerência de projetos, 
desenvolvimento, testes, controle de qualidade, responsáveis pelo suporte solicitou o desenvolvimento de um determinado projeto. Por fim o us que representava os responsáveis por fornecer as informações necessários (requis usuári correto desenvolvimento do projeto e responsáveis por utilizar o prodisitos) par vezes, clientes e usuários atuavam como as mesmas pessoas, represento gerado. À papéis simultaneamente.

Por sua vez, o estudo de Lopes et al [10] abordou a definição dos tipos de atos na engenharia de requisitos em um contexto de projetos de desenvolvimentos de atores de software. Neste caso foram considerados dois atores principais distribuid especificação e equipe de desenvolvimento. A equipe de especificacão repre equipe responsáveis pela condução da elicitação, análise, negociação, espeçifio representava os dos requisitos junto aos stakeholders, balidaça dos requisitos junto aos stakeholders, bem como a manutenção da rastreabilidade do requisitos em relação à origem. A equipe de desenvolvimento representava responsáveis pela modelagem do software com base na especificação e pela manuva os da rastreabilidade dos requisitos durante as etapas de modelagem, codificação e teste.

\subsection{Nivel de dispersão}

Em relação ao nível de dispersão, este diz respeito à distância física entre os atores envolvidos em um determinado projeto ou fase deste. O estudo realizado po Prikladnicki et al [8] alguns critérios foram definidos par classificar os niveis dispersão dos stakeholders. Estes critérios foramilos de considerandos stakeholders. Estes criterios foram utilizados em estudos de caso refinado [9] e foram de projo como atores. O estudo então foi suas características principais:

Mesma localização física: esta é a situação onde a empresa possui todos os stakeholders em um mesmo local. Nesta situação, reuniões ocorrem sem dificuldades e a equipe pode interagir estando fisicamente presente. Não existe diferença de fusohorário e as diferença culturais raramente envolvem a dimensão nacional. Os obstáculos são os já existentes no desenvolvimento centralizado de software (Figura 2).

Distância nacional: esta situação caracteriza-se por ter os stakeholders localizados dentro de um mesmo país, podendo se reunir em curtos intervalos de tempo. Dependendo do país, pode haver diferenças em relação ao fuso-horário e as diferenças culturais podem ocorrer em maior escala do que na situação anterior (Figura 3 ).

Distância continental: esta situação caracteriza-se por ter os stakeholders localizados em países diferentes, necessariamente dentro do mesmo continente. Nesta situação, as reuniões ficam um pouco mais difíceis de serem realizadas face a face, devida a distância física. O fuso-horário exerce um papel importante na equipe, podendo dificultar algumas interações (Figura 4).

Distância global: está situação caracteriza-se por ter os stakeholders localizados em países diferentes e em continentes diferentes, formando uma distribuição global. Nesta situação, reuniões face a face ocorrem geralmente no inicio dos projetos e, entre outros fatores, a comunicação e as diferenças culturais podem ser barreiras para o trabalho. O fuso-horário exerce um papel fundamental, podendo impedir interações entre as equipes (Figura 5).

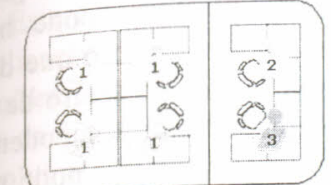

Figura 2 - Mesma localização física

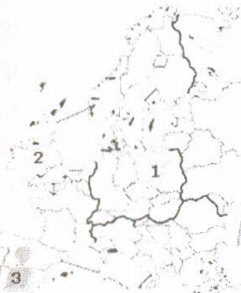

Figura 4 - Distância continenta

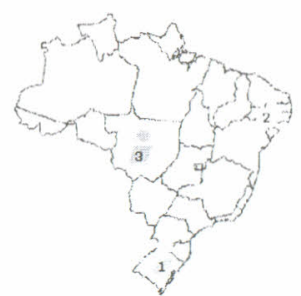

Figura 3 - Distância nacional

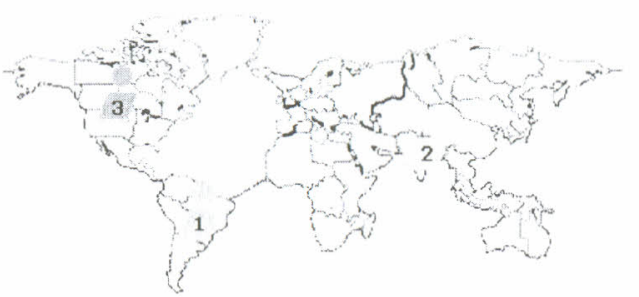

Figura 5 - Distância global

\section{Método de Pesquisa}

Esta pesquisa se caracteriza como um estudo predominantemente exploratório, sendo que o método de pesquisa principal foi o estudo de caso. Com relação à natureza do estudo, a pesquisa exploratória tem como principal finalidade desenvolver, esclarecer e modificar conceitos e idéias, com vistas à formulação de novas teorias, modelos hipóteses pesquisáveis em estudos posteriores [11].

O método de estudo de caso é adotado conforme proposto por Yin [11]. A unidade de análise foi projetos de desenvolvimento distribuído de software. Buscou-se aplicar os critérios e a classificação dos níveis de dispersão propostos. Por tratar-se de uma pesquisa qualitativa, deve-se ter claras as limitações do estudo, no que se refere ao número de organizações estudadas, restringindo a generalização dos resultados obtidos.

\section{Um modelo de classificação dos níveis de dispersão dos stakeholders}

Ao longo desta pesquisa foram encontrados alguns modelos para caracterizar o nivel dispersão geográfica em projetos de DDS [5, 12, 13]. Todos os modelos caracterizavam apenas o nível de dispersão da equipe de projeto, não considerando outros atores envolvidos no processo. Sendo assim, verificou-se uma oportunidade para refinar os modelos existentes, contribuindo no sentido de propor um modelo genérico, considerando tantos atores quantos forem necessários. Alguns refinamentos foram publicados $[8,9]$ e este estudo propõe a generalização deste modelo, de forma a classificar os níveis de dispersão de qualquer ator dentro de um projeto distribuído.

O primeiro passo a ser representado no modelo é a definição dos principais stakeholders envolvidos nos projetos. Logo após, utilizando uma representação gráfica, modelo auxilia na classificação dos níveis de dispersão, de acordo com os critérios definidos na seção 3.2. Visando uma representação fiel do nivel de dispersão entre os atores, foram definidos dois tipos de distância para a sua classificação: a distância física inter-atores e intra-atores. A distância física inter-atores representa a distância entre os atores identificados. Mas ter todos os atores distantes fisicamente de acordo com alguma 
das quatro situações previstas na seção 3.2 não indica que um determinado grupo atores esteja distribuído. Sendo assim, em um ambiente de DDS pode hapo de distribuição interna dos atores. A distância física intra-atores é o critério que defin a distância física existente dentro de uma mesma equipe (por exemplo, dentro da eque a de projeto ou do conjunto de usuários), considerando que as equipes podem equipe centralizadas ou distribuídas. No caso de distribuição, a distância física pode assumir qualquer um dos quatro critérios previstos.

\subsection{Representação do nível de dispersão}

A partir dos critérios definidos, pode-se dizer então que a caracterização de um ambiente de Desenvolvimento Distribuído de Software (DDS) ocorre sempre que pel menos um dos atores envolvidos estiver fisicamente distante dos demais. A figur propõe uma representação gráfica para definir o nível de distribuição do DDS 6 distância física inter-atores e distância física intra-atores) e a relação entre eles, considerando stakeholders genéricos.

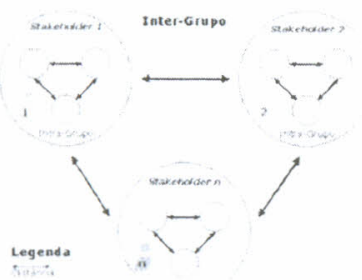

Figura 6 - Definição do nível de distribuição / dispersão no DDS

Caso existam distâncias diferentes, deve ser considerada sempre a maior distribuição, tanto intra-atores quanto inter-atores. Como exemplo, supõe-se a existência de integrantes de uma equipe de projeto nos continentes X e Y. Se o cliente estiver no continente $\mathrm{Y}$, mesmo que parte da equipe de projeto esteja no mesmo continente, outra parte estará globalmente distante (continente $\mathrm{X}$ ). Neste caso, a distância existente entre a equipe de projeto e o cliente deve ser é definida pela maior distância existente, ou seja, entre o continente $\mathrm{X}$ e o Y, caracterizando uma distância global.

Apesar de o modelo proposto considerar na sua representação apenas 3 atores, este modelo é genérico e pode ser utilizado para representar os diversos atores envolvidos. Para isso, devem ser incluídos tantos stakeholders quantos forem necessários e aplicar a correta classificação do nível de distribuição entre eles.

\section{Estudo de Caso: dois cenários de aplicação do modelo proposto}

$\mathrm{O}$ estudo de caso foi desenvolvido em uma unidade de desenvolvimento de software de uma organização de grande porte com sede nos Estados Unidos. A unidade está localizada no Rio Grande do Sul e possui em torno de 180 colaboradores trabalhando em projetos que atendem as necessidades da área de TI da empresa (demanda interna). A unidade é reconhecida como nível dois de maturidade no modelo SW-CMM desde 2003 e possui atuação em diversos países. A grande maioria dos projetos desenvolvidos envolve equipes distribuidas globalmente. Sendo assim, modelo proposto na seção 5 foi aplicado em dois cenários distintos. O primeiro cenárí diz respeito aos principais atores envolvidos em projetos de DDS. Já o segundo cenário diz respeito às atividades de engenharia de requisitos em um projeto de DDS.
Cenário

ério corresponde a um projeto cujo objetivo era o desenvolvimento de Este conara a área de manufatura da organização. Para avaliar a distribuição dos uma aplicação param definidos os seguintes atores, representados no modelo proposto stakeholders, stravés da figura 7. a equipe de projeto (P), representando todos os envolvis no atraves dimento do projeto. Seus integrantes atuavam em atividades de análise do desenvolvimentia de projetos, análise e modelagem de sistema, desenvolvimento, testes negócio, gerencia de processo; os clientes (C) representavam as pessoas físicas ou e controle de qualidatram e contrataram o projeto; os usuários (U) representavam os jurídicas que solicitaram e contrataram o projeto; os usuários (U) reprãa do software responsáveis por for responsáveis pela utilização do produto gerado.

(requisitos) e eram os restava localizada no

A equipe de projeto érios estavam localizados nos Estados Unidos, no mesmo Brasil. Os clientes e os ustarios estavam localizados nos Estados Unidos, no mestho espaço físico, mas distantes fisicamente entre si.

globalmente distante dos clientes e dos tão, podemos ampliar a visão de

De forma dividindo em mais de um tipo, como utilizado em Lopes et al [10] um dos atores dividindo representamos conforme a Figura 8.

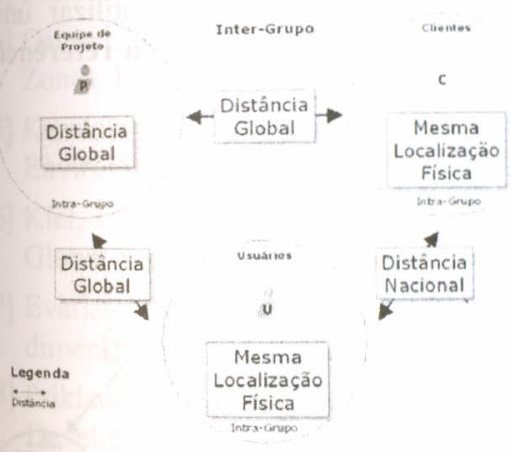

Figura 7 - Projeto 1

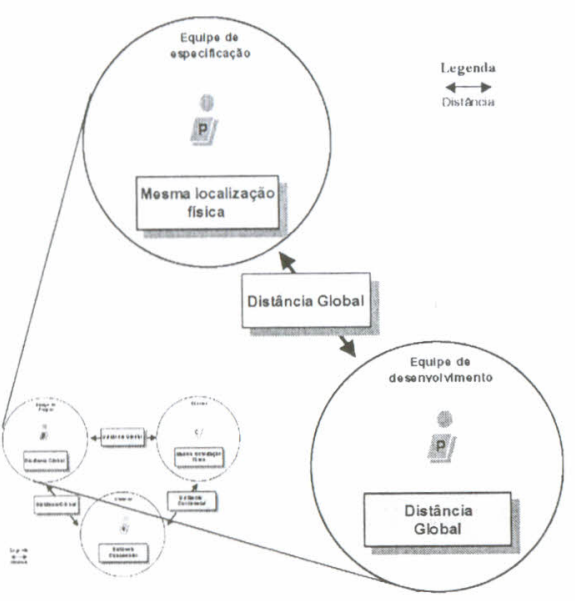

Figura 8 - Visão ampliada da equipe de projeto
Neste caso, a equipe de projeto estava dividida em equipe de especificação e equipe de desenvolvimento, com a equipe de especificação nos Estados Unidos e a equipe de desenvolvimento dispersa entre Brasil, Índia e China. Este recurso pode ser útil na representação da dispersão dos diversos papéis envolvidos no desenvolvimento de software, como testadores, analistas, entre outros.

\subsection{Cenário 2}

O cenário 2 envolvia um projeto de reengenharia de uma aplicação Web desenvolvida para gerenciar as requisições de compra de produtos da organização. A ferramenta foi projetada para auxiliar no processo de aprovação de requisições de compra e a comunicação entre compradores e fornecedores através de um workflow. O escopo do projeto inclui uma interface com o sistema de materiais onde são armazenados os itens a serem comprados e as requisições aprovadas. Melhorias da 
aplicação anterior tambem estavam incluídas no projeto. Para avaliar a distribuição stakeholders no contexto da engenharia de requisitos, foram definidos os seo do atores, representados no modelo proposto através da figura 9:

- A equipe de engenheiros de requisitos (ER) é responsável pela elicit análise, negociação, documentação, validação e gerência dos requisitos.

- O grupo de usuários (U) e clientes (C) representa quem solicito contratou o projeto, bem como os responsáveis pela utilização solicitou gerado. Esta equipe fornece informações para especificação do software.

- A equipe de desenvolvimento (D) representa as pessoas envolvid desenvolvimento de um determinado projeto, utilizando com entras no requisitos especificados pelo grupo de engenheiros de requisitos. Esta equ pode envolver gerentes de projeto, codificadores, testadores, controlado de qualidade, equipe de suporte a ferramentas, entre outros.

Os atores estavam distribuídos da seguinte forma: a equipe de engenheiros de requisitos no Brasil e nos Estados Unidos; os usuários e clientes dispersos entre Estade Unidos, Brasil, Malásia e Irlanda; a equipe de desenvolvimento estava sediada no Brasil. O grupo de usuários e clientes foi representado em conjunto, pois suas atividades no processo de engenharia de requisitos são bastante similares, fornecendo entradas para processo. Caso fosse necessário representar essa equipe em separado, poderíamos optar pela utilização de visões, como apresentado no cenário 1, ou ainda utilizar uma representação com quatro atores, como apresentado na Erro! A origem da referência não foi encontrada.

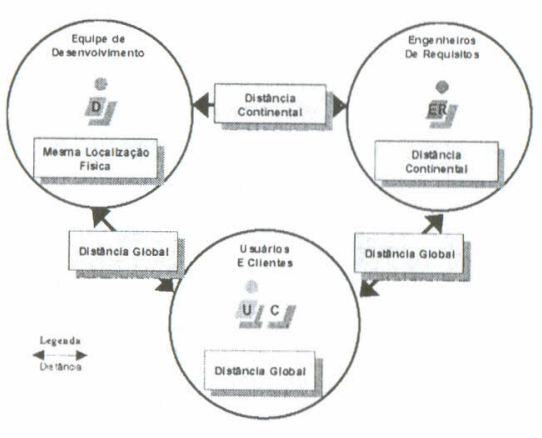

Figura 9 - Dispersão no estudo de caso 2

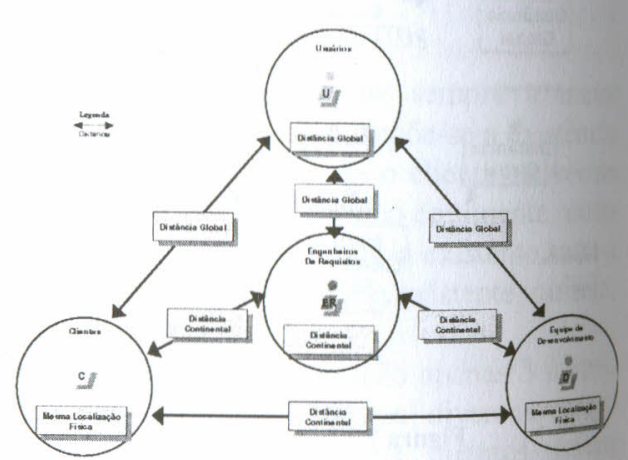

Figura 10 - Representação utilizando quatro atores

\section{Considerações finais}

Este artigo avança no conhecimento na área de DDS ao identificar critérios que permita a classificação dos níveis de distribuição em projetos de desenvolvimento distribuído. Buscou-se verificar em dois cenários a aplicabilidade do modelo proposto. Como resultado, avaliou-se que o modelo proposto permitiu a caracterização e classificação dos níveis de distribuição de cada um dos projetos estudados. Além disso, identifica-se também uma oportunidade de utilizar o modelo proposto na caracterizą̧ão e contextualização de estudos de caso desenvolvidos na área de DDS, onde é necessária a correta identificação dos atores envolvidos e dos níveis de dispersão entre eles. en melhor entendimento da área de DDS e propicia um Este comparação entre diferentes organizações, abrindo espaço para novas padrão para costa área. Devido ao restrito numero de casos estudados, os resultados pesquisas nesta a ser generalizados. Nesta fase do estudo pode-se adotar o principio da obtidos não podenalítica proposta por Yin [11]. Como contribuições deste estudo generalizaça os critérios que caracterizam os níveis de dispersão e o modelo destacam-se dito, considerando a distância física inter-grupo e intra-grupo.

propriamente dito, não se aprofundou a análise das razões que levam uma organização

Neste eścias de distribuição do seu processo de desenvolvimento de software, adotar estrategias em si. Próximos estudos nesta linha de nem o processo de de pesquisa evoluirão no sentido refinar a representação do modelo e buscar a definição de

\section{Referências Bibliográficas}

[1] Herbsleb, J. D., e Moitra, D. Global Software Development, IEEE Software Magazine, IEEE Computer Society, EUA, Mar/Abr 2001.

2] Herbsleb, J. D., Grinter, R. E. Splitting the Organization and Integrating the Code: Conway's Law Revisited. In: ICSE'99, Los Angeles, CA. Proceedings... 1999.

[3] Pressman, R. S. Software Engineering. A Practitioner's Approach. 5a edição, 2001.

[4] Carmel, E. Global Software Teams - Collaborating Across Borders and TimeZones. Prentice Hall, USA, 1999, 269p.

[5] Karolak, D. W. Global Software Development - Managing Virtual Teams and Environments. Los Alamitos, IEEE Computer Society, USA, 1998, 159p.

[6] Kiel, L. Experiences in Distributed Development: A Case Study, In. Workshop on Global Software Development at ICSE, Oregon, EUA. Proceedings... 2003.

[7] Evaristo, Roberto; Scudder, Richard. Geographically distributed project teams: a dimensional analysis. In: HICSS, 2000, Havaí. Proceedings... EUA, p. 1-15, 2000.

[8] Prikladnicki, Rafael; Audy, Jorge Luis N.; Evaristo, Roberto. Distributed Software Development: Toward an understanding of the relationship between project team, users and customers. In: ICEIS, 2003, Angers. Proceedings... França, p. 417-423, Abr. 2003

[9] Prikladnicki, R. MuNDDoS: Um Modelo de Referência para Desenvolvimento Distribuído de Software. 2003. Dissertação de Mestrado, PPGCC - PUCRS, 2003.

[10]Lopes, Leandro; Prikladnicki, Rafael; Majdenbaum, Azriel; Audy, Jorge. "Uma proposta para processo de requisitos em ambientes de desenvolvimento distribuido de software". In: 6th WER, Piracicaba. Brasil. Proceedings... 2003. p. 329-342.

[11]Yin, R. K. Case study research: design and methods, Sage, USA, 1994

[12]Espinosa, J. Alberto; Carmel, Erran. Modeling coordination costs due to tim separation in Global Software Teams. In: Workshop on Global Software Development at ICSE, 2003, Oregon. Proceedings... EUA, p. 64-69, 2003.

[13]Paasivara, Maria Communication needs, practices and supporting structures in global inter-organizational software development projects. In: Workshop on Global Software Development at ICSE, 2003, Oregon. Proceedings... EUA, p. 59-63, 2003. 